NOTAS E INFORMAÇŌES

NOTES AND INFORMATIONS

\section{SOBRE A PRESENCCA DE ARTÉRIA FACIAL EM CAPRINO}

\section{FREDERILO UZHNAM LARREIRU E SILUH Professol Hdiunto} Universidade Federal de Uberlandia

PEURU PRIMO HOMBONATU Professor Assistente Doutor Zootecnia da USP

LELSO RLUES RODRIGUES

Assistente nIvel 10

Centro de Ciências Haráias e Veterinarias de Laqes

RENATO SOUTO SEVERINO Professor Adjunto

Universidade Federal de Uberlandia

ANDRE LUIS QUAGLIATO DOS SANTOS Professor Hssistente Universidade Federal de Uberlandia Faculuade de Medicina Veterinaria e

INTRODULAO

- comportamento anatomico do sistema circulatorio constitui oonto de reparo a ser considerado pelos pesauisadores, uma vez que os tratados de Anatomia veterinaria objetivam somente os aspectos de normalidade anatomica, fi cando, às nossas expensas, as descricas das variacós e casulsticas cansideravelmente frequentes e importantes. 0 fato dessas variaças serem numerosas tem nos levado a uma analise mais minuciosa do comportamento vascular, atingindo diretamente nossa relacar quando do proprio ensino do sistema aos alunos dos cursos de graduacao. De sorte que, nă têm sido raras as vezes em que temos tido oportunidade de identificar as tais modificacoes quando do preparo de material para as aulas praticas, alertandonos assim, para a constante necessidade de revisão dos ensinamentos, valendo-nos da comunicacăo das referidas variacós. Assim, o objetivo deste trabalho e a indicacăo e a descricăo de variacaes no comportamento das arterias responsaveis, em parte, pela irrigacaro dos planos superficiais da regiăo facial em caprinos, mais especificamente na presenca de uma arteria facial que determina modificaças substanciais quanto ao comportamento de vasos contiguos que tambem colaboram na nutricăo da citada regi a.

A consulta aos classicas livros de Anatomia evidencia, num breve relato, unanimidade no registro da ausência de uma arteria facial em pequenos ruminantes. conferindo a diferentes vasos a irrigacăo da regiao que, em outras es pecies animais, cabe a arteria facial.

\section{MATERIAL E METODO}

GUES, C.A.; SEVERINO, R.5.; SANTOS, A.L.Q. Sobre a presenca de arteria faciab em caprino. Rev. Fac. Med. Vet. Zootec. Univ. S. Paulo, $25(2): 309-315,1988$

RESUMO: Os autares observaram na hemiface direita de um caprino SRD a presenca da arteria facial que, nessa especie animal, usualmente esta ausente. 0 comDortamento e a distribuifaro da citada artéria, bem como as alteraços por ela determinadas no esquema vascular da reairo săo descritas pelos autores.

UNI TERMO5: Anatomia, caprinos; Artéria facial
0 presente trabalho baseia-se no encontro de variacăo nao relatada, ou seja, a presenca de uma arteria facial em caprino adulto sem raca definida. A peca examinada e componente de material preparado para as tarefas praticas dos alunos de graduacăo da Universidade Federal de Uberlandia-MG. Assim, as animais foram abatidos nos proprios da citada Escola, onde tambem foi injetado com solucao aquosa de formol a $10 \%$ e posterior fixacao oor imersao na mesma solucão. Para melhorar o procadimeat de fixacao, valemo-nos da introduca da soluca pelas arterias carbtidas comuns direita e esaueraa. Na cabeca que. mediante dissectao tos olanos superficiais, notamos comoortamento vascular diferente do usual, procedemos a injecao do sistema circulatbrio arterial. atraves da abicacao de Neoprene latex "450" corado nas arterias carotidas 
comuns direita e esquerda. Essa tecnica foi precedida de lavagem com massagem do sistema com solucaro fisiologica a temperatura ambiente, buscando eliminar possiveis grumos causados peio fixador. Essa cabeca, assim preparada, foi submetida a uma disseccaro mais apurada, tendo-se tomado fotografias e confeccionado esquemas da distribuicaro arterial dos planos superficiais da regia facial da hemiface direita que apresentou tal variacåa.

\section{RESULTADOS}

Para os trabalhos praticos do curso de Veterinaria da Universidade Federal de Uberlândia, foram dissecadas 20 hemicabecas de caprinos adultos SRD, tendo-se encontrado em 19 hemifaces, distribuicao arterial conforme descricgo classica da literatura. No entanto, em uma hemicabeca, correspondendo à hemiface direita de um caprino macho, encontramos um calibroso ramo arterial que acompanha o trajeto da veia facial. Apos disseccalo da origem e distribuiça da referida arteria, notamos que ela possui comportamento semelhante ao da arteria facial de outras especies de animais, mais particularmente, dos bovinos.

A arteria facial, como designamos na peca em apreco, origina-se, por um tronco comum com a arteria lingual, da artéria carotida comum direita, formando assim, na sua emergencia, um tronco linguofaciab que mede cerca de $1,6 c m$ e tem aproximadamente $0,4 \mathrm{~cm}$ de calibre.

Assim, o referido tronco linguofacial surge da face ventral da arteria carotida comum direita, correndo medialmente ao ventre caudal do músculo digastrico para, afinal, enviar um delgado ramo que nasce de sua face caudal. Da face rostral surge outro ramo que se destina à musculatura do palato mole e tecidos adjacentes, ao osso estilohiobide, para em seguida terminar constituindo, lateralmente, a arteria facial e, medialmente, a arteria lingual.

A arteria facial, assim originada, cruza o tenda comum aos ventres do musculo digastrico, caudalmente ofereiendo a esse nlvel um ramo que se destina à glândula salivar mandibular. A seguir atinge a borda caudal do múscula masseter, emitindo, a 1 , outro vaso para a glandula mandibular. Continua em trajeto roztralmente, relacionando-se com a boral ventral do musculo masseter, para - qual envia, de sua face dorsal, dois jequenos colaterais. Da faca ventral notamos a origem de dois pequenos colaterais aue se dirigem rostralmente ate atingir o musculo depressor do baio mandibular. Esses vasas relacionam-se com a veia facial e o ramo bucal ventral

Rev. Fac. Med. Vet. Zootec. Univ. S. Paulo, 25(2):309-315, 1988. do nervo facial, lateralmente, com o ducto parotideo, dorsaimente. Finalmente, da origem a dois ramos arteriais enderecados ao musculo masseter e termina por oferecer a arteria labial mandibular, ventralmente, e arteria ladial maxilar, dorsalmente.

Nesse caso, a arteria transversa da face que e responsavel em parte pela sua vascularizacăo, acha-se reduzida a um vaso de pequeno calibre e extensao que se restringe, provavelmente, as camadas superficiais da regiao maxilar.

\section{COMENTARIOS E CONCLUSOES}

A consulta aos livros classicos de Anatomia denotam a existencia de três tipos de relatos para o comportamento dos vasos arteriais responsaveis pela irrigaço superficial da regiăo facial dos pequenos ruminantes.

Un primeiro grupo de autores, como ZIMMERL, 8 (1930); 8055 I et alii, 1 (s.d.); GONZALEZ y GARCIA \& GONZALEZ v ALVAREZ, 4 (1961), nao explicita as diferencas entre os bovinos e os pequenos ruminantes, afirmando laconicamente que a presenca da arteria facial ou artéria maxilar externa é observada nos ruminantes como sendo ramo da arteria carbtida externa, considerando-a como dividida numa porfăo maxilar e noutra facial, nå se detendo na descriça das regiaes de vascularizaça, tampouco indicando claramente se a mesmo comportamento pode ser observado nos pequenos ruminantes. A nosso ver, o relato, como pode ser evidenciado em outros capltulos, foi embasado no comoortamento dos vasos da regiao dos bovinos, nao podendo ser considerado para os pequenos ruminantes.

Num segundo grupo de autores, como MAY, 5 (1964) e ELLENBERGER \& BAUM, 2 (1932), notamos descriçes que nos levam a acreditar ser a arteria transversa da face, o vaso responsavel pela nutricao da regiao estudada, visto que os citados autores identificam as arterias labial maxilar e labial mandibular como ramos da arteria transversa da face e, tambem, como sendo os vasos que irrigam a regiao em apreco, năo fazendo mença à existência ou nåo de uma arteria facial nos pequenos ruminantes.

- terceira grupo, camo NICKEL et alii, 7 (1981); MONTANE \& BOURDELLE, 5 (1917); GETTY, 3 (1981), assinala que o tronco linguofacial, assim como a arteriafacial, estao ausentes nos pequenas ruminantes. Para esses autores, a arteria lingual surge diretamente da arteria carotida externa, sendo que a area de irrigacaro da arteria facial a assumida pela arteria transuersa aa face, nessas especies. Assim, ooservando a aescriczo que merecem os ramos da arteria trans- 


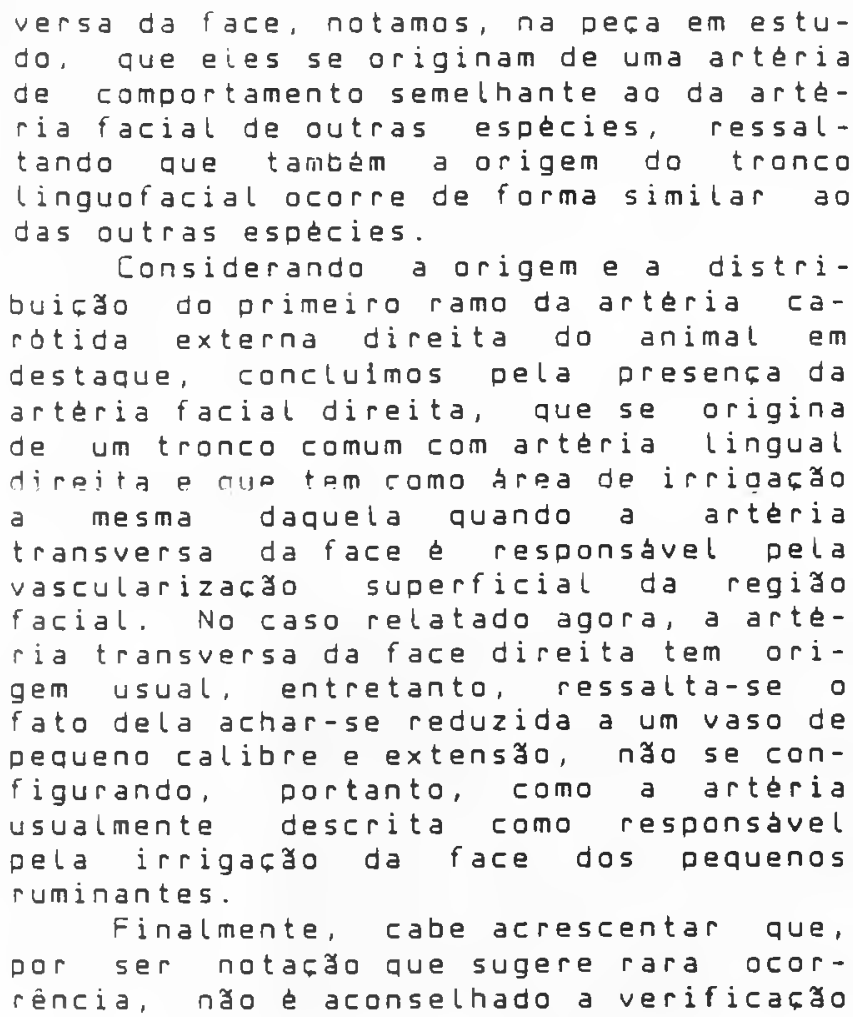

da frequência do fato, tampouco analise estatistica.

SILUA, F.O.C.: BOMBONATO, P.P.; RODRIGUES, C.A.; SEVERINO, R.S.; SANTOS, A.L.Q. About the occurrence of the facial artery in the caprine. Rev. Fac. Med. Vet. Zootec. Univ. 5. Pauln. $25(2) \cdot 309-315 \quad 1988$

SUMMARY: The AR. reported the occurrence of the right facial artery a half-face of caprine. According to literature, the facial artery is absent in the $5 \mathrm{mall}$ ruminants. The traject, distribution and pattern of the facial artery has been related by the AR.

UNITERMS: Anatomy of goats; Facial arteries 


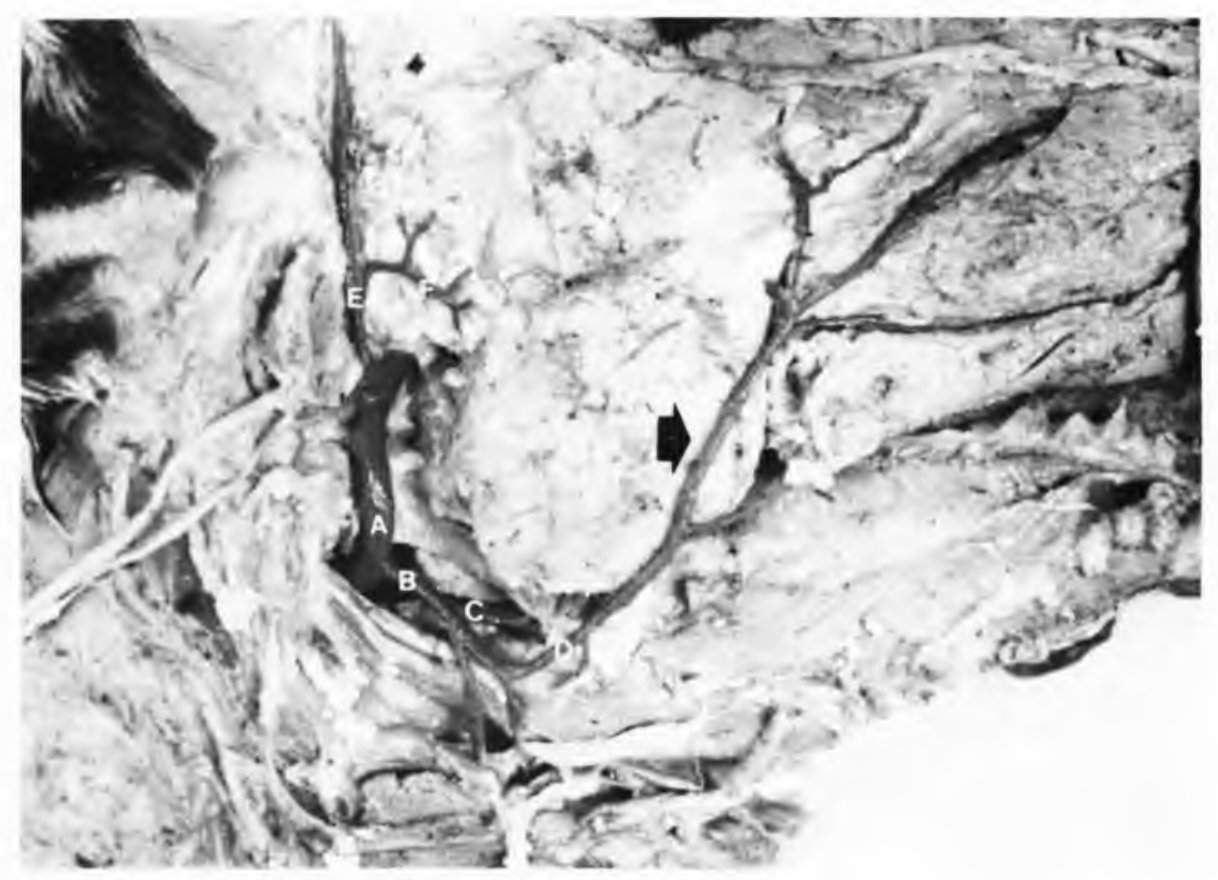

FIGURA 1 - Fotografia de parte da hemiface direita de caprino, mostrando a artéria carótida externa direita (A) fornecendo o tronco linguofacial (B) que, por sua vez, oferece as artérias lingual (C) e facial (D) (seta). Nota-se, ainda, a artéria temporal superficial (E) dando origem à artéria transversa da face (F) que, neste caso, acha-se reduzida em tamanho e calibre. 
- BOSSI, V.; CARADONNA, G.B.; ZIMMERL, U. Trattata di anatomia veterinaria. Milano, Francesco vallardi, s.d. v.2, p.161.

2 - ellengerger, W. \& BRUM, H. Handbuch तer veraleichenden Anatomie der Haustiere. 17. Auf. Berlin, Julius Springer, 1932. p. 665.

3 - GETTY, R. Sisson and Grossman's the anatomy of the domestic animals. 5.ed. Philadelphia, W.B. Saunders, 1981. v.1, p. 1006.

4 - gonzalez y GARCia, J.\& GONZALEZ y ALVAREZ, R. Anatomia de las animales domesticos. 7 .ed. Madrid, Grafica Canalis, 1961. p. 683.
5 - MAY, N.D.S. The anatomy of the sheep. 2.ed. 5t. Lucia, University of dueensland, 1954. 0 . 153.

6 - montane, L. \& gourdelle, e. Anatomie regionale des animaux domestiques. Paris, J.-B. Baililiere et Fils, 1917. v.2, D. 104 .

7 - NICKEL, R.; SCHUMMER, A.; SEIFERLE, $E$. The anatomy of the domestic animals. Berlim, Paul Parey, 1981. จ. Э, ค. 103 .

8 - ZIMMERL, U. Trattato di anatomia veterinaria. Milano, Francesco vallardi, 1930. v.2, p. 124.

Recebido para publicacgo em $10 / 11 / 87$ Aprovado para publicaça em 5/5/88 